\title{
Research on Designment of Management System for Power Source Equal- izing Charge Based on FPGA control
}

\author{
Haifeng Lin and Ruili Mao*
}

Beijing Information Technology College, Beijing

\begin{abstract}
The accumulator can store the energy in high capacity, and the super capacitor can charge and discharge in high power. The mixed power source composed by the accumulator and super capacitor not only has the characteristics for both of them but also meets the high-power requirement of high capacity and peak value. How to perform the equalizing charging for multiple power packs is a emphasis in the industry currently. On the basis of analysis for multiple equalizing charging methods, a new type of design scheme based on DC/DC and switch matrix is raised in this project, the thinking of intermittent charging mode is adopted and four BCAP0350 is served as the charging sample to perform the charging and discharging experiment as well as verify the composite charging design scheme is provided with the feasibility.
\end{abstract}

Keywords: DC/DC Type, Switch Matrix, Equalizing Charge, Intermittent charging.

\section{INTRODUCTION}

With rapid development of such electronic technical devices as electronic instrument equipment, digital mobile terminal and electrodynamic force locomotive, especially for putting into operation of some electronic equipment used for loading, since they are provided with such characteristics as high peak power and low average power, namely, the higher power output is required to be provided during peak value. Currently, the power density of accumulator employed in the market is low, and the charging and discharging are slow. If the demand of high current output is required to be met, the accumulator is required to be provided with high capacity, and this kind of power source may increase the equipment loading. As a new type of energy storage element, the supercapacitor is provided with such advantages [1] as rapid charging and discharging, long service life, high power density, strong service environment adaptability, safety and no toxicity, these advantages is very applicable for pulsatility loading, however, the energy density is low so that the traditional accumulator can't be replaced to individually supply the power for loading. The common type currently is that such characteristics as high energy density of accumulator and high power density of supercapacitor are combined to design a kind of mixed power source to improve the output power of power peak value and reduce the volume and mass of power source for putting into operation.

However, the rated voltage of mixed power source for single module is $3.2 \mathrm{~V}$ so as not to meet the output demand of high-voltage equipment, therefore, this kind of mixed power source is required to conduct the series-parallel connection so as to meet the power supply demand of equipment. While the excessive charging and discharging of monomer in the combined power source may shorten the service life of power pack and even produce the explosion so as to threaten the equipment safety. The primary cause for the excessive discharging of monomer is the capacity difference among power source of each monomer inside power pack. One of methods to solve this problem is equalizing charge, currently; main study emphasis is on such two parts: firstly, design for equalizing charge circuit topology; secondly, study on equalizing control strategy.

On design of equalizing charge circuit topology, the basis is weather the energy consumption may be divided into energy consumption type and non-energy consumption type, therein, the energy consumption type is based on the resistance discharging equalizing method for heat dissipation and energy consumption of equalizing resistance; nonenergy consumption type is based on switch capacitor method, switch inductance method, DC/DC method and multiwinding transformer method [2]. There are such problems as long equalizing time and short universality for common equalizing method currently; meanwhile, the consistency based on external pressure of accumulator is adopted to judge the factor that the instability exists for equalizing. How to rapidly and effectively perform the equalizing charge for single power source inside power pack is an important study direction in the industry.

Beginning from the super-capacitorin this project, the series connection of four mixed power sources is served as the study object, and based on DC/DC and by utilizing the switch matrix, the composite design thinking of multiequalizing charge is adopted to put forward the intersected charging design concept, design a kind of power source management system, establish the mathematical model on charging and discharging as well as design a kind of composite rapid charging method so as to demonstrate the feasibility of system existence via the simulation and experiment. 


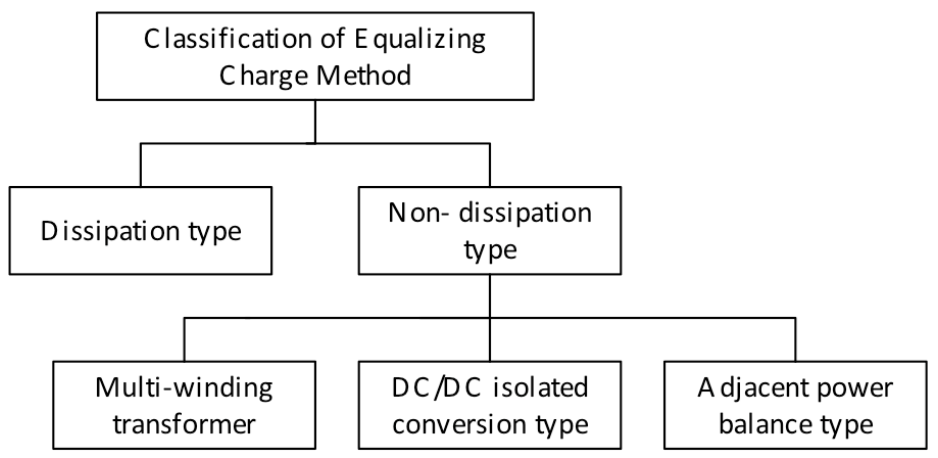

Fig. (1). Category on Common Equalizing Charge Method Currently.

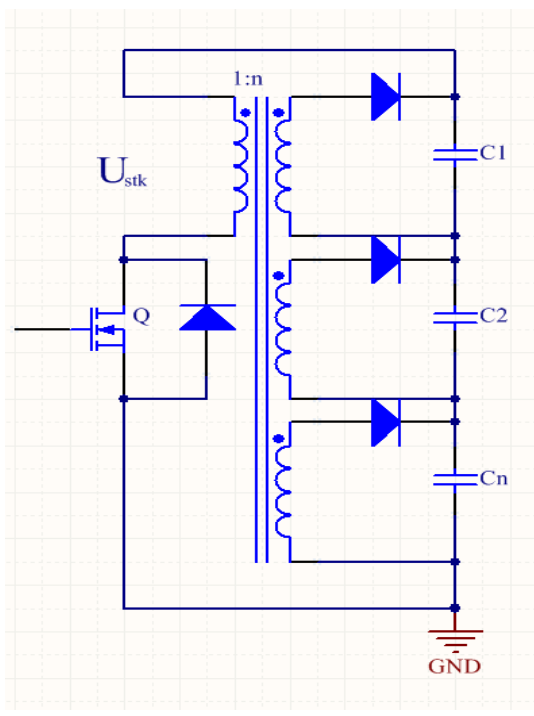

Fig. (2). Schematic Diagram of Multi-winding Transformer Method.

\section{INTRODUCTION TO EQUALIZING CHARGE METHOD}

As shown in Fig. (1), the common equalizing charge method may be divide into energy dissipation type and nonenergy dissipation type base on the energy dissipation, therein, the equalizing with energy dissipation type is to discharge the monomer accumulator with high capacity respectively through shunting the resistance in parallel on both ends of each monomer power source in the power pack and through shunting the resistance until the capacity of all monomer accumulators is on same level. The design of this circuit is simple and the cost is low, however, the shunting resistance may be in working status to consume up the energy of monomer power source in the mode of heat [3] to be applicable for the occasion with sufficient energy and good heat dissipation generally. The non-dissipation equalizing circuit is studied mainly in this project, the common non-energy dissipation equalizing circuit covers the following kinds.

\subsection{Multi-Winding Transformer Method}

The principle of multi-winding transformer method is as shown in Fig. (2), only a transformer is required for realization, with convenient design and simple principle. The primary edge of transformer is connected with input; each auxiliary edge is respectively connected with a charging receptor, in the design of transformer, provided that the number of turns of winding for each auxiliary edge is guaranteed to be same, the voltage of auxiliary edge may be guaranteed to be same [4]. When unequalizing condition occurs for monomer power source, $\mathrm{Q}$ breakover and the energy storage of transformer are realized. After QSTK cuts off, the energy is coupled onto the auxiliary edge. If the voltage of power source monomer is lower than USTK/n, the power source monomer shall be connected with the diode of monomer torque, the auxiliary edge of transformer releases the energy towards this monomer, the lower of terminal voltage, the more of energy absorbed by power source monomer. In the process of actual application, especially when there are more power source monomers required to be charged, the transformer structure may be more complex, meanwhile, the cost is higher for realization of complete consistency for winding of auxiliary edge, what's more, the mutual inductance matching among winding and the magnetic bias of magnetic core for transformer may cause instable charging and produce certain difficulty on application. 


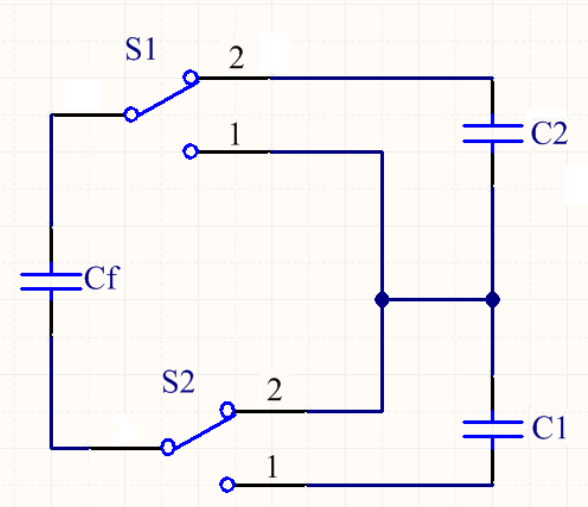

Fig. (3). Principle Diagram of Switch Capacitor Method.

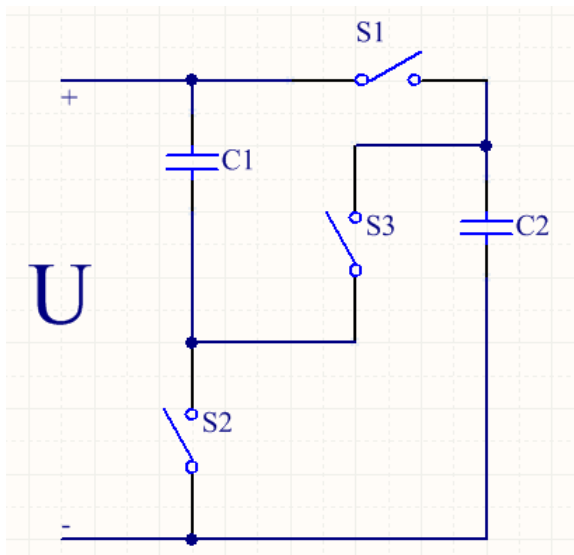

Fig. (4). Charging Method with Series-Parallel Conversion.

\subsection{Switch Capacitor Method}

Adjacent power source equilibrating type mainly means that the capacitor is served as the energy storage element to be served as the bridge of energy transfer to complete the energy equilibrium among each monomer power source inside power pack. The principle is as shown in Fig. (3), each capacitor acts with two adjacent monomer power sources to convey the energy from high-voltage monomer to voltage monomer. Therein, $\mathrm{Cf}$ is the transferred capacitance, $\mathrm{S} 1$ and S2 are placed Location 2, C2 may discharge towards Cf due to higher voltage, the voltage for both of them becomes equal nearly. Above-said action is conducted for many times to realize the transfer of electric energy on $\mathrm{C} 1$ and $\mathrm{C} 2$ so as to reach the purpose of voltage equilibrating [5]. The insufficiency for this kind of scheme is because that slow equilibrium speed, complex circuit and characteristics of multiway switch can't enable the complete equilibrium between two monomer power sources appropriately. On the other hands, the individual circuit parameter required to be designed in detail is required for configuration of different power source systems, the parameter selection is difficult, and the research and development is difficult.
Except that the capacitor is utilized to transfer the energy, and the inductance is also utilized to transfer the energy. The further introduction will not be conducted due to limit of paper length.

\subsection{Charging Method with Series-Parallel Conversion for Switch Matrix}

The principle of this method is as shown in Fig. (4), indicating that multiple power source monomers in series converts it as the parallel form via switch matrix while charging so as to guarantee the individually charge for each power source body simultaneously, and the series form is changed after completing the charging to be used for discharging. While charging, close $S_{1}$ and $S_{2}$ as well as open $S_{3}$ so as to enable that monomer power source $C_{1}$ and $C_{2}$ work under parallel status. After completing the charging, disconnect $S_{1}$ and $S$ as well as close $S_{3}$ so as to enable that monomer power source $\mathrm{C}_{1}$ and $\mathrm{C}_{2}$ work under series status. The principle structure for this kind of method is simple so as to realize the deep charging and discharging, however, in the actual application, the electrotechnics difficulty exists, only being applicable for the condition that the number for monomer power 


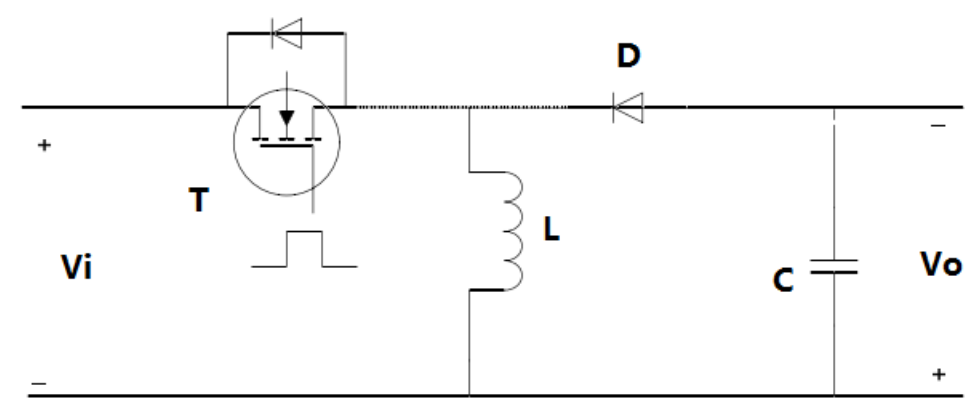

Fig. (5). Schematic Diagram of Boost-Buck DC/DC Convertor.

source in series is less. When the number for monomer power source in series is more, the quantity of switch is huge extremely, and the wiring may be very complicated.

\subsection{DC/DC Method}

$\mathrm{DC} / \mathrm{DC}$ convertor is adopted with power tube as the connection between the front end and back end, via the way of digital control, to achieve the control of the DC voltage in the back end by controlling the breaking time of power tube with "chopping" signal. The item is taken with FPGA as the main control chip, to monitor the voltage, current and other signals in real time by it, and adjust the duty ratio to output stable DC voltage according to the monitoring value. The DC/DC convertor is usually divided into boost, buck and boost-buck according to function. We will make study and discussion to the boost-buck DC/DC convertor [6].

The schematic diagram of boost-buck circuit is as shown in Fig. (5), which not only can be used as step-down circuit but also can be used as boosted circuit, among them, $\mathrm{L}$ is inductance, D is a one-way connected diode, $\mathrm{C}$ is capacitance, $T$ is a power tube, when the control end of $T$ is input with the full impulse voltage, $\mathrm{T}$ is forward connected, and cut off when it is zero. At the stage of connecting of power tube, the input current returns after passing $\mathrm{T}$ and $\mathrm{L}$ due to the existence of $\mathrm{D}$, at this moment $\mathrm{VL}=\mathrm{Vi}$, the current of inductance is increasing step by step. When by the moment of $\mathrm{t} 1, \mathrm{~T}$ is switched off, input voltage $\mathrm{Vi}$ and the back end is switched off, the current of $\mathrm{L}$ returns through $\mathrm{D}$ after passing the load, at the same time the current of capacitor $\mathrm{C}$ returns to negative terminal through the load. The current of inductance is decreasing gradually, and the voltage is in opposite direction, with the inductance as the energy source, this moment inductance voltage $\mathrm{V}_{\mathrm{L}}=\mathrm{V}_{\mathrm{o}}$.

At the stage of connecting of power tube, inductance current:

$i_{L}=\frac{\int_{0}^{t_{1}} V_{L} d t}{L}=\frac{\int_{0}^{t_{1}} V_{i} d t}{L}=\frac{V_{i} \times t_{1}}{L}$

In Switching off of power tube, inductance current:

$$
i_{L}=\frac{\int_{t_{1}}^{T} V_{L} d t}{L}=\frac{\int_{t_{1}}^{T} V_{o} d t}{L}=\frac{V_{o} \times\left(T-t_{1}\right)}{L}
$$

According to current balance principle:

$\frac{V_{O}}{V_{\text {in }}}=\frac{t_{1}}{T-t_{1}}=\frac{D}{1-D}$

Among them $D=t_{1} / T$ is the duty ratio.

Above formulas show that the specific value of input voltage and output voltage can be adjusted by the duty ratio of input square wave, that is to achieve the constancy of output voltage by controlling the square wave inputting of control end of DC/DC circuit according to the change of input voltage.

The principle of charging method of DC/DC is as shown in Fig. (6), which is constant-voltage charged by parallel charging monomer of $\mathrm{DC} / \mathrm{DC}$ module, when the voltage of power source monomer is lower than rated value, DC/DC module will charge it. When the voltage value reaches the rated voltage, close the DC/DC module. The system power source can charge several power source monomers at the same time by this method, with high accuracy, small consumption, high charging speed, but the DC/DC module must be matched with the power source monomers one by one, when the power source is made up of several monomers, the overall charged circuit system will be very large, complex, and the cost will be very high [7].

\section{A MODIFIED NEW AND EQUALIZING CHARG- ING CIRCUIT DESIGN}

Compared with the above equalizing charging circuits, in view of their advantages, a new and equalizing charging circuit with FPGA as the core, based on the switch matrix and $\mathrm{DC} / \mathrm{DC}$ module is designed, this circuit is only adopted with one $\mathrm{DC} / \mathrm{DC}$ module, and is charged circularly by the switch matrix. The using of only one DC/DC module guarantees the accuracy of equalizing charging, meanwhile the circuit cost 


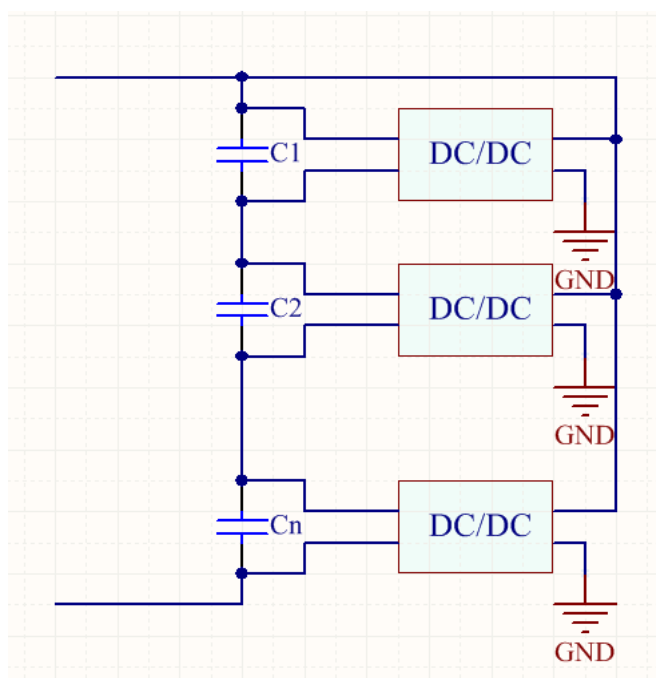

Fig. (6). Block Diagram for Charging Principle of DC/DC.

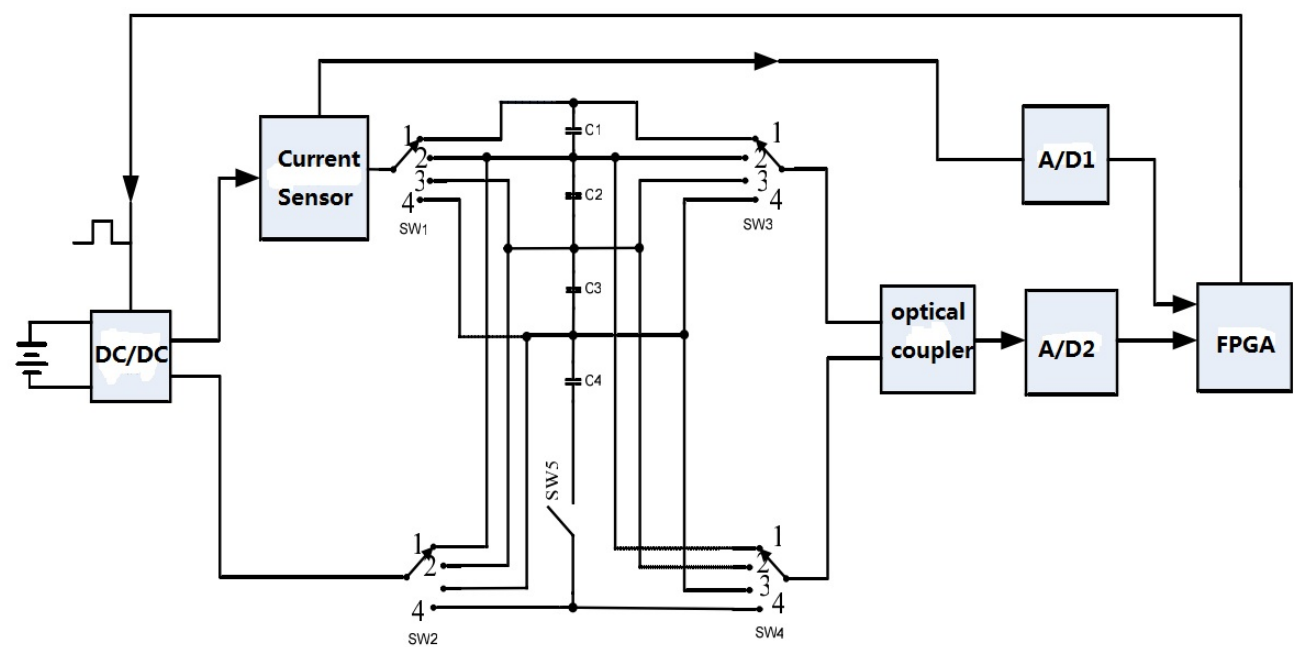

Fig. (7). Block Diagram of Combined Type Equalizing Charging Design Principle.

is reduced, but this method is limited by work of the switch matrix, is not suitable for excessive capacitor charging, if several super-capacitors charging is required, they can be grouped to use this method, and then parallel mode will be used to carry out equalizing charging between the groups.

The system principle is as shown in Fig. (7), which is composed of charging module (on the left) and monitoring module (on the right). The specific work procedure of the system is as follows: FPGA carry out waveform control to DC/DC module, to output the power source of each monomer to charge after voltage transformation, the charging target is selected by FPGA controlling switch matrix. The switch matrixes consist of SW1, SW2, SW3, SW4, single blade four set switch and a single blade switch SW5, when charged, switch off the SW5 first, when the four single blade four set switches are at position of "1", the DC/DC module will charges the monomer power source 1 . Similarly, the monomer power source 2, 3, 4 can be charged respectively, after completion of charging, four single blade four set switches are switched off, SW5 is closed. FPGA monitors the charging current in real time by AD1, and controls the DC/DC module and switching network based on the sampling value. In the process of charging, the system monitors the voltage of power source monomer in real time, and the monitoring value couples the data to $\mathrm{AD} 2$ by optocoupler, and sent to FPGA for analysis after sampled, and control the switch matrix, to charge the module 1, 2, 3, 4 of monomer power source respectively [8].

\section{IMPROVEMENT OF WAY OF INTERRUPTING METHOD CHARGING IN VIEW OF SUPER CAPAC- ITOR}

The traditional charging methods are frequently adopted with two stage charging style of "the constant electric current and the constant voltage", this method can avoid impact 


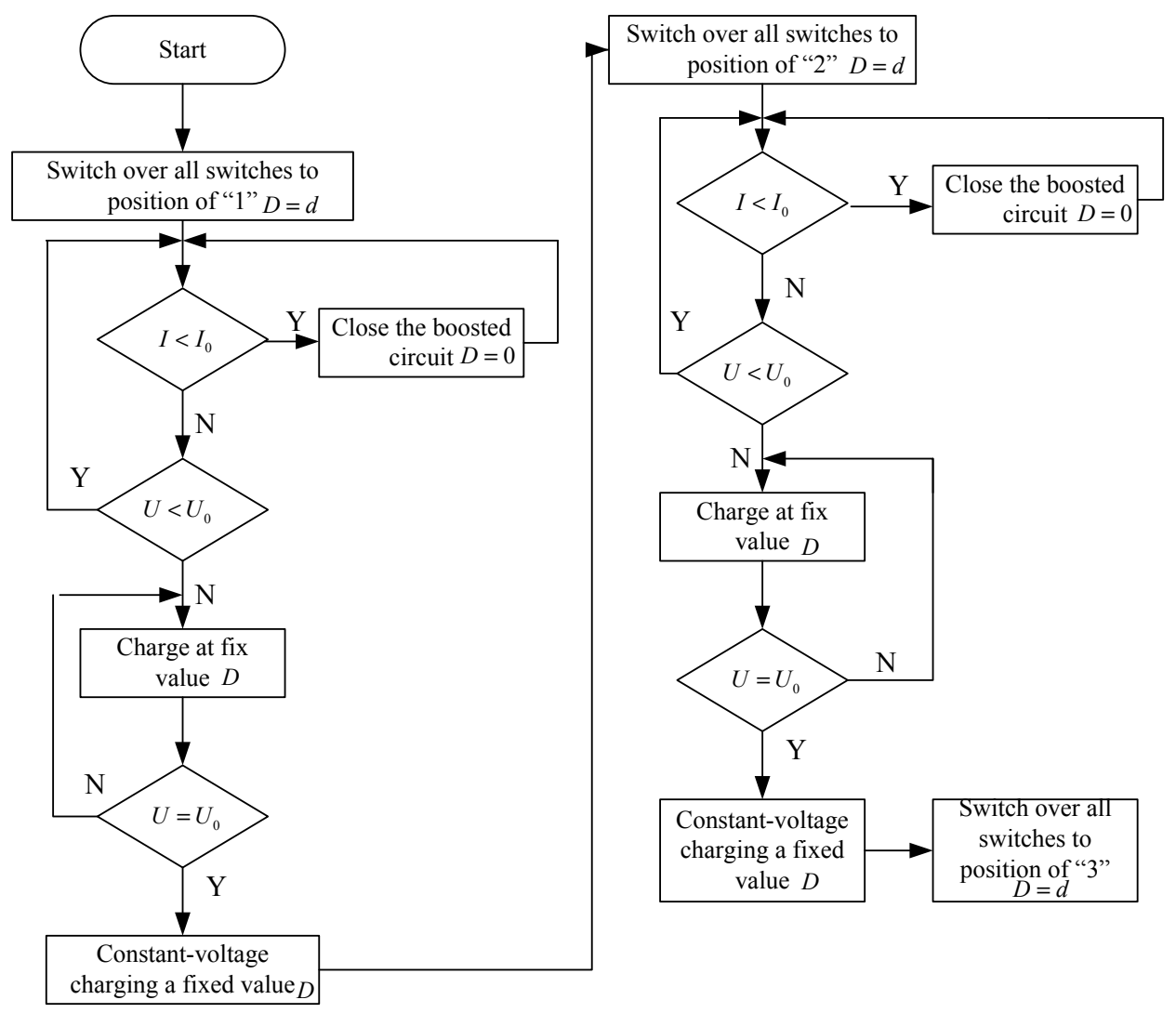

Fig. (8). FPGA Control Flow Diagram.

of peak current to monomer power source and DC/DC circuit, for protection of the equipment. The constant current mode is used in the first stage, the voltage of single power source is increasing gradually with the time, when the voltage of single power source reaches a certain value, changes over to constant voltage mode, the charging current is reducing gradually, until is fully charged. This item carries out segment treatment for first stage charging process, to use interrupted method to charge, which not only protects the super capacitor in the single power source, but also causes no obvious effect on charging time. The specific working principle is as below: FPGA samples the system voltage and current by $\mathrm{A} / \mathrm{D}$, when the current value is too big, the FPGA chip will close the control end of DC/DC module for several periods, this moment the DC/D module is in the state of not working, then the charging current is reducing rapidly, the single voltage value of super capacitor is not increasing longer, then FPGA chip open the switch, and continue to control the PWM end, thus the charging current can be maintained constant basically, after the voltage of power source monomer is increasing to a certain value, the charging mode is changed to constant-voltage charging, that is to fix the square wave duty ratio of PWM control end of DC/DC module, until the single power source is charged to the rated voltage, to complete the charging of the power source monomer, and then switch over the position of the switch, charge the next power module [8].
According to the charging methods, the control procedure of FPGA is as shown in Fig. (8), when starting the system, all the switches are placed at position of "1" to conduct charging control to the super capacitor C1, PWM end of $\mathrm{DC} / \mathrm{DC}$ circuit is adopted with the waveform with duty ratio $\mathrm{D}=\mathrm{d}$ to charge, when inspecting that the charging current is too big, close the PWM end, this moment $\mathrm{D}=0$, the charging current is reducing, when charging current I is reducing below the required current, open the control of PWM end to continue to carry out the $\mathrm{D}=\mathrm{d}$ charging mode, until the voltage value of super capacitor $\mathrm{C} 1$ is charged to the required value, and then charge with constant voltage method, that is $\mathrm{D}$ is a fixed value, charged to the rated voltage, then switch over the switch to the next super capacitor monomer, roundrobin like this, finally finish the equalizing charging of the whole super capacitor unit.

\section{SUMMARY AND ANALYSIS}

This item is combined with several mature equalizing charging methods to put forward a equalizing charging design thought based on switch matrix and DC/DC module and set up the related model and real system according to the design thought, the charging process is adopted with disconnected charging method, to guarantee the work of super capacitor within the rated current, the simulation of the charging process is as shown in Fig. (9). 

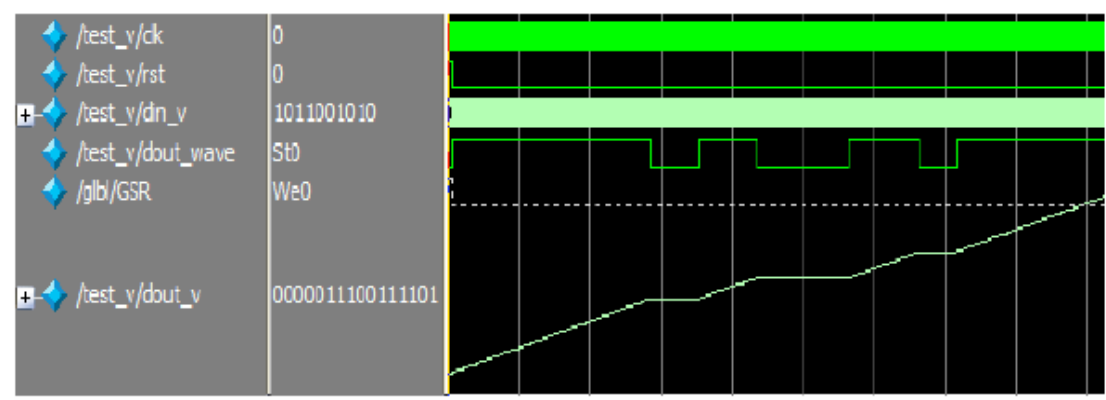

Fig. (9). Simulation Effect of Disconnected Charging.

Table 1. Measuring Value Of Charging Result.

\begin{tabular}{|c|c|c|c|c|c|c|c|c|c|c|}
\hline & I & II & III & IV & V & VI & VII & VIII & IX & $\mathbf{x}$ \\
\hline \hline $\mathrm{C} 1$ & 2.68 & 2.69 & 2.73 & 2.73 & 2.76 & 2.71 & 2.71 & 2.69 & 2.72 & 2.68 \\
\hline $\mathrm{C} 2$ & 2.71 & 2.72 & 2.72 & 2.72 & 2.69 & 2.73 & 2.70 & 2.71 & 2.71 & 2.70 \\
\hline $\mathrm{C} 3$ & 2.69 & 2.72 & 2.71 & 2.68 & 2.72 & 2.70 & 2.70 & 2.69 & 2.68 & 2.69 \\
\hline C4 & 2.75 & 2.74 & 2.77 & 2.73 & 2.73 & 2.75 & 2.74 & 2.73 & 2.69 & 2.71 \\
\hline \multirow{2}{*}{ Capacity Deviation } & 0.07 & 0.05 & 0.06 & 0.05 & 0.07 & 0.05 & 0.04 & 0.04 & 0.04 & 0.03 \\
\cline { 2 - 18 } & $97.4 \%$ & $98.2 \%$ & $97.8 \%$ & $98.2 \%$ & $97.5 \%$ & $98.2 \%$ & $98.5 \%$ & $98.5 \%$ & $98.5 \%$ & $98.9 \%$ \\
\hline
\end{tabular}

The item is used four BCAP0350 super capacitor of Maxwell as the charging monomer samples, the rated voltage is $2.7 \mathrm{~V}$, and rated capacity is $350 \mathrm{~F}$. use this method to charge, after completion of balancing, the voltage values of the four capacitor monomers are as shown in Table 1, the maximum error of the monomer in the same group is $0.07 \mathrm{~V}$, the purpose of equalizing charging is reached basically.

It is observed from the simulation and validation results of this item that the $\mathrm{DC} / \mathrm{DC}+$ switch matrix mode is used to reducing the $\mathrm{DC}$ conversion module and increase the switch matrix network, when the number of the power monomers in power group is increasing, the cost of complexity of the switch matrix will increase, the system design method set forth in the item can be applied in the working environment with few number of monomers, for case of big number of monomers, the way of multiple groups paralleling can be used. How to balance the charging time of the monomers in the group and number of monomer grouping is the research direction in next stage.

\section{CONFLICT OF INTEREST}

The authors confirm that this article content has no conflicts of interest.

\section{ACKNOWLEDGEMENTS}

This work is supported by the Importation and Development of High-Caliber Talents Project of Beijing Municipal Institutions.

\section{REFERENCES}

[1] D. Wang, "Study on Energy Storage System Control Strategy of Vehicle Super Capacitor Based on Power Distribution," Beijing Jiaotong University. 2011

[2] B. Dong, and Y. Li, "Storage Battery Capacity Balancing Method Overview," Journal of Power Source, no. 5, 2011.

[3] J. Li, "Research and Implementation of Li-Ion Power Battery Management System," Zhejiang Science and Technology University 2010.

[4] X. Shi, "Parameter Test and Balancing Technology Research of Parallel Super Capacitor Pack," Harbin Institute of Technology, 2006.

[5] R. Lu, T. Wang, C. Zhu, and Q. Wang, "Dynamic Balance Control Algorithm of Super Capacitor Pack Based on Fit Capacitor," Journal of Harbin Institute of Technology, no. 9, 2008.

[6] Y. Liu, "Design of Protective Circuit in BUCK type DC-DC Convertor," Hunan University, 2010.

[7] G. Chen, "Two-way Equalizing Battery of Electric Vehicles Management System Design," Hunan University, 2011.

[8] S. Feng, Q. Liu, and Z. Li, "Design and Implementation of Super Capacitor Equalizing Charging Circuit Based on FPGA," Power Source Technology, no. 5, 2011.

(C) Lin and Mao; Licensee Bentham Open.

This is an open access article licensed under the terms of the Creative Commons Attribution Non-Commercial License (http://creativecommons.org/licenses/by$\mathrm{nc} / 3.0 /$ ) which permits unrestricted, non-commercial use, distribution and reproduction in any medium, provided the work is properly cited. 